Journal of Computer Science 6 (4): 428-438, 2010

ISSN 1549-3636

C 2010 Science Publications

\title{
Integrity Classification Algorithm of Images obtained from Impact Damaged Composite Structures
}

\author{
Mahmoud Z. Iskandarani \\ Department of Electrical Engineering, Faculty of Engineering, Al-Zaytoonah University of Jordan, \\ P.O. Box 911597, Post Code 11191, Amman, Jordan
}

\begin{abstract}
Problem statement: Many NDT systems used for damage detection in composites are difficult to apply to complex geometric structures, also, they are time-consuming. As a solution to the problems associated with NDT applications, an intelligent analysis system that supports a portable testing environment, which allowed various types of inputs and provided sufficient data regarding level of damage in a tested structure was designed and tested. The developed technique was a novel approach that allowed locating defects with good accuracy. Approach: This research presented a novel approach to fast NDT using intelligent image analysis through a specifically developed algorithm that checks the integrity of composite structures. Such a novel approach allowed not only to determine the level of damage, but also, to correlate damage detected by one imaging technique using available instruments and methods to results that would be obtained using other instruments and techniques. Results: Using the developed ICA algorithm, accurate classification was achieved using CScan and Low Temperature Thermal imaging (LTT). Both techniques agreed on damage classification and structural integrity. Conclusion: This very successful approach to damage detection and classification is further supported by its ability to correlate different NDT technologies and predict others.
\end{abstract}

Key words: Image processing, NDT, composites, impact damage, algorithm, modeling

\section{INTRODUCTION}

Carbon fiber composites are now fairly widely used in civilian and military applications. Delaminations are common defects found in these materials. Their presence leads to structural weaknesses, which cause failure of used components. It is important to develop effective nondestructive testing procedures to identify these defects and to increase the safety early enough to avoid catastrophic failure. Hence, all methods for fast and reliable inspection deserve special attention (Wolf et al., 2004; Gupta and Breitenstein, 2007; Gralewicz and Owczarek, 2005; Bohm et al., 2006; Montanini and Aliquo, 2009; Shah et al., 2006; Colvin, 2005; Tohgo et al., 2009).

Composite materials depend on their structural arrangement to obtain their desired mechanical properties. The fibers are generally of little practical use but with a well designed combination of fibers and matrix a reliable component with optimal performance is produced. Integrity of a material is based on quality of fabrication as the designed specifications are not only determined by the best available structural properties, but also any other combination of properties for a particular application. So, it is important to be able to tailor and optimize the manufacturing process with tools able to inspect defects that most often can have a marked effect on the component performance and functionality. Two main areas of concern in inspection and damage classification:

- During manufacturing

- In-service

A developed inspection technique and classification algorithm should cover two main areas:

- Critical damage identification and detection

- Analysis and monitoring of damage progression

Impact damage is a key issue in the design of composite structures where the impact event and extent are of importance. Damage occurs progressively during an impact and is a function of the impact event and structure resistance that is affected by material properties. Local and global effects need to be considered which gives an indication regarding the structure dynamic response. Method of impacting is also a factor where supported frames respond differently to impact compared to unsupported ones, 
indicating that boundary conditions significantly affect structure response and extent of damage.

The application of an impact can result in a dynamic stress which when established can induce a damage that propagates at a number of sites within the material thickness. Composites with their low transverse tensile strength can be prone to this type of effect.

Under normal conditions, material constituents in a structure are bound to their respective potential levels with relative stability. As impact energy is applied, shock waves (impulses) may cause damage such as fiber breakage or cracks (that can propagate over time). When a defect is induced, the original energy distribution would be affected, hence, new energy levels and pockets of energy sub-levels will be formed. This energy re-mapping can be correlated to the applied force of impact and classified through the developed classification system (Mouritz et al., 2009; Li et al., 2009; Breitzman et al., 2009; Kim et al., 2009; Hayman, 2007; Stoika et al., 2009)

Predictive and classification analysis is an important tool. In developing such system (Zangani et al., 2007; Williams et al., 2008; Zhang and Richardson, 2004; Goebel et al., 2006a; 2006b; Hu et al., 2006; Eklund and Goebel, 2005; Verdegaya et al., 2008; Chinnam and Baruah, 2007; Jenab and Rashidi, 2009), certain things have to be considered to enable criteria for classification and ranking for tested structures:

- Defects arrangement on the basis failure contribution

- Tested components arrangement in terms of a theoretical possibility of failure in certain applications

- Untested components arrangement in terms of their tendency to fail in certain applications

- Classification of known test results into categories

- Classification model development

- Manufacturing and testing process modification based on the established model

- New components test and model modification

- Use of probability to predict defect effect on component failure

In this study a novel classification algorithm based on searching an image for pixel re-distribution is used for damage classification. The proposed technique is suitable for high volume monitoring and inspection of safety critical components non-destructively.

\section{MATERIALS AND METHODS}

Figure 1a represents a model of a damaged composite sample. The Fig. 1 illustrates directions of wave travels through the tested sample once exposed to a testing source. Such signals are captured and analyzed by the developed ICA algorithm below.

Integrity Classification Algorithm (ICA): This novel approach to defect detection is based on wavelength and color intermixing as illustrated in the following steps:

- Before format conversion, the captured image is allocated a map as shown in Fig. 1b

- The primary colors for each obtained image are intermixed according to and the results are stored and then converted to data using a purposely developed search through algorithm

- The conversion of a searched through image will result in a text file that holds the necessary information regarding the state of the tested component

- All resulted data files per image are then divided into strings of data each as a sequence stored in a specific file using our developed Matrix-Column Algorithm (MCA) before being correlated to produce a decision regarding component health

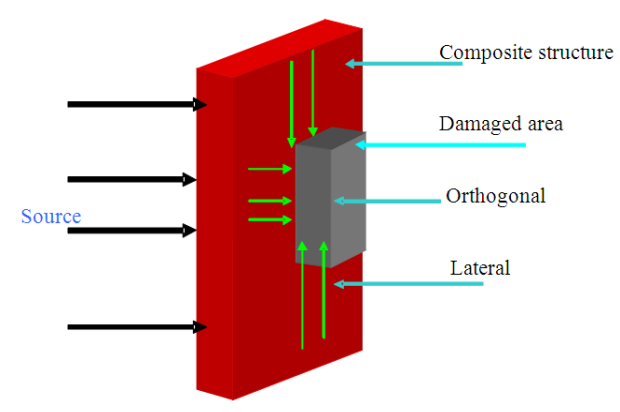

Fig. 1a: Model for a damaged sample under test

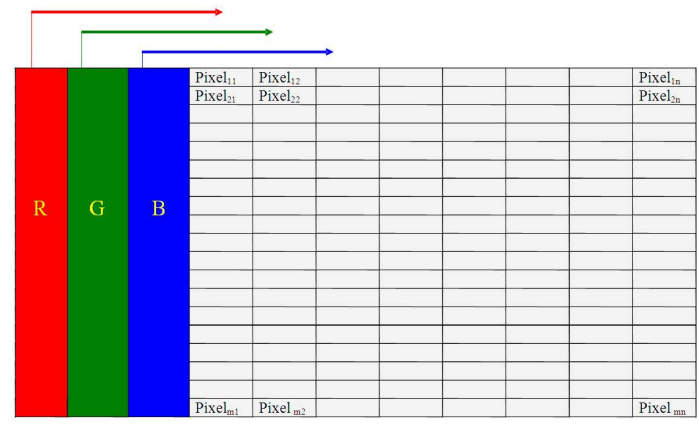

Fig. 1b: Image color intermixing 
In the MCA algorithm, the converted image is filtered into sequences $S_{1}-S_{m}$ containing vectors of individual column matrices extracted from the converted source image data file. The overall extracted matrix consists of discrete combination of all column sequences as in Eq. 1:

$$
\left[\begin{array}{lcc}
\mathrm{S}_{1} & \mathrm{~S}_{2} & \mathrm{~S}_{\mathrm{m}} \\
\mathrm{a}_{11} \cdot \mathrm{r}_{11} & \mathrm{a}_{12} \cdot \mathrm{r}_{12} & \mathrm{a}_{1 \mathrm{~m}} \cdot \mathrm{r}_{1 \mathrm{~m}} \\
\mathrm{a}_{21} \cdot \mathrm{r}_{21} & \mathrm{a}_{22} \cdot \mathrm{r}_{22} & \mathrm{a}_{2 \mathrm{~m}} \cdot \mathrm{r}_{2 \mathrm{~m}} \\
& & \\
& & \\
\mathrm{a}_{\mathrm{n} 1} \cdot \mathrm{r}_{\mathrm{n} 1} & \mathrm{a}_{\mathrm{n} 2} \cdot \mathrm{r}_{\mathrm{n} 2} & \mathrm{a}_{\mathrm{nm}} \cdot \mathrm{r}_{\mathrm{nm}}
\end{array}\right]
$$

Where:

$\mathrm{a}_{\mathrm{ij}}=$ Original matrix elements

$\mathrm{r}_{\mathrm{ij}}=$ Amplitude factor

From (1) we obtain:

$S_{1}=\left[\begin{array}{l}A_{1} \\ A_{2} \\ A_{n}\end{array}\right], S_{2}=\left[\begin{array}{l}B_{1} \\ B_{2} \\ B_{n}\end{array}\right] \ldots, S_{m}=\left[\begin{array}{l}X_{1} \\ X_{2} \\ \\ X_{n}\end{array}\right]$

From (2) we obtain:

$\mathrm{S}_{1 \mathrm{~T}}=\sum_{\mathrm{i}=1}^{\mathrm{n}} \frac{\mathrm{A}_{\mathrm{i}}}{\theta}, \mathrm{S}_{2 \mathrm{~T}}=\sum_{\mathrm{i}=1}^{\mathrm{n}} \frac{\mathrm{B}_{\mathrm{i}}}{\theta}, \ldots \mathrm{S}_{\mathrm{mT}}=\sum_{\mathrm{i}=1}^{\mathrm{n}} \frac{\mathrm{X}_{\mathrm{i}}}{\theta}$

where, $\theta$ is a normalizing factor.

As the original matrix is simplified in (3), the data classification column matrix is represented in Eq. 4:

$$
\mathrm{D}=\left[\begin{array}{l}
\mathrm{S}_{1 \mathrm{~T}} \\
\mathrm{~S}_{2 \mathrm{~T}} \\
\\
\mathrm{~S}_{\mathrm{mT}}
\end{array}\right]
$$

The designed classification algorithm takes into account reference, undamaged sample images in its operations to achieve a decision regarding structure integrity as a function of required application. Considering Eq. 4, the required decision function can be derived as follows.

For a reference sample image, (4) becomes:

$$
\mathrm{D}_{\text {Ref }}=\left[\begin{array}{l}
\mathrm{S}_{\text {ITref }} \\
\mathrm{S}_{2 \text { Tref }} \\
\\
\mathrm{S}_{\mathrm{mTref}}
\end{array}\right]
$$

With a damaged sample image given by:

$$
\mathrm{D}_{\text {Dam }}=\left[\begin{array}{l}
\mathrm{S}_{\mathrm{ITDam}} \\
\mathrm{S}_{2 \mathrm{TDam}} \\
\\
\mathrm{S}_{\mathrm{mTDam}}
\end{array}\right]
$$

For damaged/undamaged decision and using (5) and (6) we obtain:

$$
R=\left[\begin{array}{l}
\left|S_{1 T R e f}-S_{\text {TTDam }}\right| \\
\left|S_{2 \text { Ref }}-S_{2 \text { TDam }}\right| \\
\left|S_{\text {mTRef }}-S_{\text {mTDam }}\right|
\end{array}\right]
$$

The resulting values are substituted in a predecision matrix P:

$$
\mathrm{P}=\left[\begin{array}{l}
\lambda_{1} \\
\lambda_{2} \\
\\
\lambda_{\mathrm{m}}
\end{array}\right]
$$

For a perfectly undamaged composite structure, $\mathrm{P}=0$. For damaged components, each element in $\mathrm{P}$ contributes by its values to the overall classification of level of damage. Using Eq. 3 and 8, we obtain:

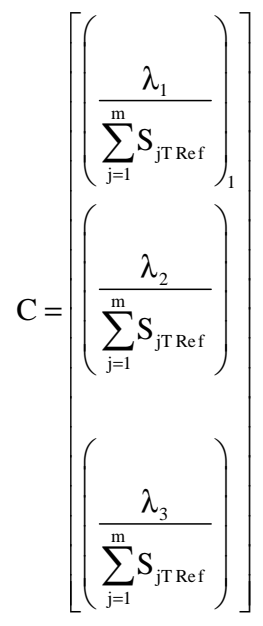


For practical applications, each element in Eq. 9 should not exceed a certain value $\beta$ for the component to be acceptable. Hence, the final decision per testing technique is based on matrix $\mathrm{F}$ given by:

$$
\mathrm{F}=\left[\begin{array}{l}
\beta_{1} \\
\beta_{2} \\
\beta_{\mathrm{m}}
\end{array}\right]
$$

For an over all correlated decision, a hybrid matrix is used as in Eq.11:

$$
\mathrm{F}_{\mathrm{Hybrid}}=\left[\begin{array}{llll}
\beta_{11} & \beta_{21} & \ldots & \beta_{\mathrm{n} 1} \\
\beta_{12} & \beta_{22} & \ldots & \beta_{\mathrm{n} 2} \\
& & & \\
& & & \\
\beta_{\mathrm{m} 1} & \beta_{\mathrm{m} 2} & \ldots & \beta_{\mathrm{mn}}
\end{array}\right]
$$

\section{RESULTS}

Figure 2 and 3 show C-Scan images obtained for $5 \mathrm{~mm}$ Woven Glass before and after an impact at $28.6 \mathrm{~J}$, while Figure 4 and 5 show Low Temperature Thermal imaging (LTT) for the same component. Table 1 and 2 show the MCA algorithm results for C-Scan and LTT images, while Table 3 and 4 show same results as in Table 1 and 2 but re-grouped to show the MCA results before impact and after impact for both C-Scan and LTT.

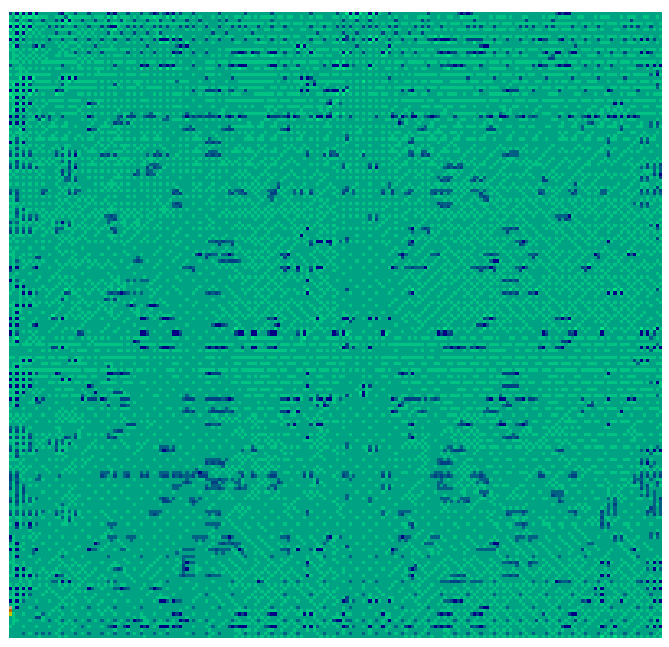

Fig. 2: Pre-Impact C-Scan image for $5 \mathrm{~mm}$ woven glass component

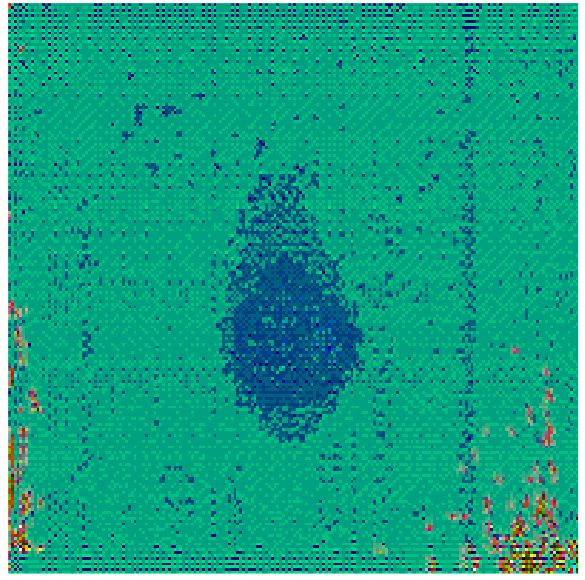

Fig. 3: Post-impact (28.6 J) C-Scan image for $5 \mathrm{~mm}$ woven glass component

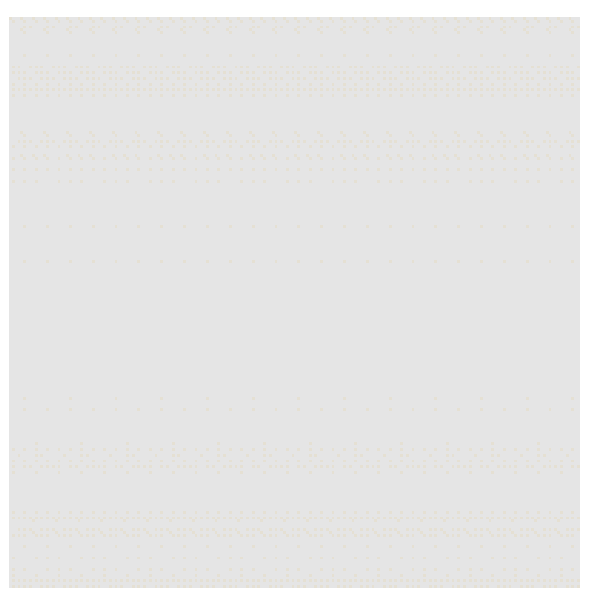

Fig. 4: Pre-impact LTT image for $5 \mathrm{~mm}$ woven glass component

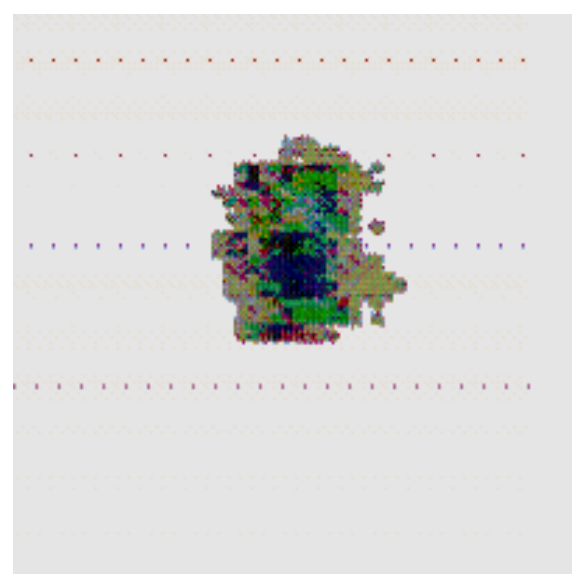

Fig. 5: Post-impact (28.6 J) LTT image for $5 \mathrm{~mm}$ woven glass component 
J. Computer Sci., 6 (4): 428-438, 2010

\begin{tabular}{|c|c|c|c|c|c|c|c|c|c|}
\hline$\overline{S_{1} \text { Ref }}$ & $\mathrm{S}_{1 \mathrm{Dam}}$ & $\mathrm{S}_{2 \text { Ref }}$ & $\mathrm{S}_{2 \mathrm{Dam}}$ & $\mathrm{S}_{3 \mathrm{Ref}}$ & $\mathrm{S}_{3 \mathrm{Dam}}$ & $S_{4 \text { Ref }}$ & $\mathrm{S}_{4 \mathrm{Dam}}$ & $\mathrm{S}_{5 \mathrm{Ref}}$ & $S_{5 \mathrm{Dam}}$ \\
\hline 0 & 7 & 0 & 37 & 0 & 0 & 0 & 8 & 0 & 0 \\
\hline 0 & 21 & 0 & 14 & 0 & 0 & 0 & 1 & 0 & 0 \\
\hline 0 & 0 & 1250 & 2831 & 0 & 0 & 0 & 0 & 0 & 0 \\
\hline 578 & $\begin{array}{r}0 \\
602\end{array}$ & 0 & 0 & 0 & 0 & 0 & 2 & 0 & 0 \\
\hline $\begin{array}{l}578 \\
0\end{array}$ & 623 & 0 & 32 & 0 & 2 & 1 & 4 & 0 & 0 \\
\hline 0 & $\begin{array}{l}0 \\
0\end{array}$ & $\begin{array}{l}0 \\
0\end{array}$ & 36 & 0 & 0 & 0 & 1 & 0 & 0 \\
\hline 0 & 1 & $\begin{array}{l}0 \\
1\end{array}$ & 0 & $\begin{array}{l}0 \\
0 \\
0\end{array}$ & 6 & $\begin{array}{l}0 \\
0\end{array}$ & $\begin{array}{l}0 \\
0\end{array}$ & $\begin{array}{l}0 \\
0\end{array}$ & $\begin{array}{l}0 \\
0\end{array}$ \\
\hline 0 & 1 & 0 & 19 & 0 & 0 & 0 & 5 & 0 & 12 \\
\hline 0 & 1 & 0 & 12 & 0 & 5 & 0 & 0 & 0 & 0 \\
\hline 0 & 7 & 0 & 0 & 0 & 4 & 0 & 0 & 0 & 1 \\
\hline 0 & 0 & 0 & 0 & 0 & 1 & 0 & 3 & 0 & 1 \\
\hline 0 & 0 & 0 & 0 & 0 & 4 & 0 & 5 & 0 & 0 \\
\hline 0 & 0 & 0 & 0 & 0 & 0 & 0 & 0 & 0 & 0 \\
\hline 0 & 10 & 0 & 0 & 0 & 5 & 0 & 0 & 0 & 1 \\
\hline 0 & 0 & 0 & 0 & 0 & 6 & 0 & 0 & 0 & 0 \\
\hline 0 & 12 & 0 & 0 & 0 & 0 & 0 & 4 & 0 & 21 \\
\hline 0 & 0 & 0 & 0 & 0 & 2 & 0 & 1 & 0 & 0 \\
\hline 0 & 6 & 26969 & 23306 & 0 & 4 & 0 & 0 & 0 & 0 \\
\hline 0 & 11 & 0 & & 0 & 1 & 0 & 0 & 0 & 0 \\
\hline 0 & 0 & 0 & 172 & 0 & 2 & 0 & 5 & 0 & 0 \\
\hline 0 & 17 & 0 & 125 & 0 & 0 & 0 & 8 & 0 & 0 \\
\hline 0 & 39 & 0 & 0 & 0 & 4 & 0 & 0 & 0 & 1 \\
\hline 0 & 10 & 0 & 24 & 0 & 0 & 0 & 0 & 0 & 0 \\
\hline 0 & 3 & 2 & 18 & 0 & 0 & 0 & 0 & 0 & 0 \\
\hline 0 & 0 & 0 & 10 & 0 & 1 & 0 & 0 & 0 & 0 \\
\hline 0 & 8 & 10102 & 8785 & 0 & 5 & 0 & 0 & 0 & 0 \\
\hline 0 & 4 & 0 & 0 & 0 & 2 & 0 & 0 & 0 & 0 \\
\hline 0 & 0 & 0 & 129 & 0 & 0 & 0 & 0 & 0 & 0 \\
\hline 0 & 10 & 0 & 61 & 0 & 0 & 0 & 0 & 0 & 0 \\
\hline 1 & 10 & 0 & 0 & 0 & 23 & 0 & 1 & 0 & 0 \\
\hline & 5 & 0 & 25 & 0 & 12 & 0 & 0 & 0 & 0 \\
\hline 0 & 0 & 0 & 14 & 0 & 0 & 0 & 5 & 0 & 14 \\
\hline 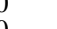 & 0 & & & 0 & 3 & 0 & 4 & 0 & 0 \\
\hline J & 0 & 152 & $10 / 3$ & 1 & 14 & 0 & 0 & 0 & 0 \\
\hline 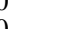 & 0 & 0 & 0 & 0 & 14 & 0 & , & 0 & 7 \\
\hline , & 0 & 0 & 21 & 0 & 0 & 0 & 19 & 0 & 0 \\
\hline 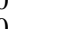 & 0 & 0 & 14 & 0 & 0 & 0 & 9 & 0 & 0 \\
\hline 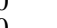 & 0 & 0 & 0 & 0 & 0 & 0 & 2 & 0 & 6 \\
\hline 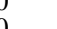 & 0 & 0 & 2 & 0 & 0 & 0 & 0 & 0 & 4 \\
\hline 0 & $\begin{array}{l}1 \\
0\end{array}$ & 0 & 6 & 0 & $\begin{array}{l}0 \\
0\end{array}$ & $\begin{array}{l}0 \\
0\end{array}$ & $\begin{array}{l}0 \\
8\end{array}$ & $\begin{array}{l}0 \\
0\end{array}$ & $\begin{array}{l}5 \\
0\end{array}$ \\
\hline 0 & 3 & 0 & 1 & 0 & 0 & 0 & 0 & 0 & 2 \\
\hline 1 & 2 & 0 & 0 & 0 & 0 & 0 & 4 & 0 & 0 \\
\hline 0 & 0 & 0 & 0 & 943 & 1906 & 0 & 2 & 0 & 0 \\
\hline 0 & 2 & 0 & 0 & 0 & 0 & 0 & 6 & 0 & 1 \\
\hline 0 & 2 & 0 & 0 & 0 & 27 & 0 & 0 & 0 & 1 \\
\hline 0 & 4 & 0 & 0 & 0 & 21 & 0 & 0 & 0 & 23 \\
\hline 0 & 5 & 0 & 1 & 0 & 0 & 0 & 0 & 0 & 0 \\
\hline 0 & 0 & 0 & 1 & 0 & 0 & 0 & 0 & 0 & 18 \\
\hline 0 & 0 & 0 & 0 & 0 & 0 & 0 & 0 & 0 & 37 \\
\hline 0 & 0 & 0 & 0 & 0 & 0 & 0 & 0 & 0 & 7 \\
\hline 0 & 0 & 0 & 0 & 0 & 0 & 0 & 0 & 0 & 2 \\
\hline 0 & 0 & 0 & 0 & 0 & 0 & 0 & 0 & 0 & 2 \\
\hline 0 & 0 & 0 & 0 & 0 & 0 & 0 & 0 & 0 & 11 \\
\hline 580 & $\begin{array}{l}0 \\
825\end{array}$ & 0 & $\begin{array}{r}0 \\
\end{array}$ & $\begin{array}{r}0 \\
944\end{array}$ & $\begin{array}{r}0 \\
8080\end{array}$ & 0 & $\begin{array}{r}0 \\
120\end{array}$ & 0 & 5 \\
\hline
\end{tabular}

\section{DISCUSSION}

From Table 1-4 and Fig. 6-9, the following is realized:

- The difference in pixel occupation between pre and post damaged images

- The difference in pixel concentration between CScan and LTT images for pre and post impact damaged components
- The ability to construct a full spectrum of sequences that each set $\left[S_{1}, \ldots S_{m}\right]$ represents a type of image resulted from a different NDT testing technique and as some techniques are better in detecting certain defects than others, Intelligent algorithms can be used to predict the response using a technique from the response of others

- It is easily noticeable from applying the above expressions that C-Scan Complements LTT and can be used as a second confirmation to the state of the tested structure 
J. Computer Sci., 6 (4): 428-438, 2010

Table 2: LTT sequences for $5 \mathrm{~mm}$ woven glass

\begin{tabular}{|c|c|c|c|c|c|c|c|c|c|}
\hline$S_{1 \text { Ref }}$ & $\mathrm{S}_{1 \mathrm{Dam}}$ & $\mathrm{S}_{2 \text { Ref }}$ & $\mathrm{S}_{2 \text { Dam }}$ & $\mathrm{S}_{3 \mathrm{Ref}}$ & $\mathrm{S}_{3 \mathrm{Dam}}$ & $\mathrm{S}_{4 \mathrm{Ref}}$ & $\mathrm{S}_{4 \mathrm{Dam}}$ & $\mathrm{S}_{5 \mathrm{Ref}}$ & $\mathrm{S}_{5 \mathrm{Dam}}$ \\
\hline$\overline{0}$ & 0 & 0 & 26 & 0 & 0 & 0 & 47 & 0 & 0 \\
\hline 0 & 4 & 0 & 16 & 0 & 0 & 0 & 7 & 0 & 0 \\
\hline 0 & 0 & 0 & 0 & 0 & 0 & 0 & 27 & 0 & 0 \\
\hline 0 & 0 & 0 & 1 & 0 & 0 & 0 & 0 & 0 & 0 \\
\hline 0 & 19 & 0 & 2 & 0 & 2 & 0 & 122 & 0 & 0 \\
\hline 0 & 0 & 0 & 0 & 0 & 0 & 0 & 98 & 0 & 0 \\
\hline 0 & 0 & 0 & 0 & 0 & 32 & 0 & 0 & 0 & 0 \\
\hline 0 & 73 & 0 & 0 & 0 & 39 & 0 & 94 & 0 & 0 \\
\hline 0 & 29 & 0 & 0 & 0 & 0 & 0 & 103 & 0 & 0 \\
\hline 0 & 58 & 0 & 0 & 0 & 29 & 0 & 6 & 0 & 0 \\
\hline 0 & 0 & 0 & 0 & 0 & 18 & 0 & 0 & 0 & 0 \\
\hline 0 & 0 & 0 & 0 & 0 & 1 & 0 & 0 & 0 & 0 \\
\hline 0 & 0 & 0 & 0 & 0 & 3 & 0 & 0 & 0 & 5 \\
\hline 0 & 0 & 0 & 0 & 0 & 0 & 0 & 0 & 0 & 0 \\
\hline 0 & 1 & 0 & 7 & 0 & 21 & 0 & 0 & 0 & 2 \\
\hline 0 & 0 & 0 & 0 & 0 & 8 & 0 & 0 & 0 & 4 \\
\hline 0 & 12 & 0 & 1 & 0 & 0 & 0 & 0 & 0 & 1 \\
\hline 0 & 0 & 0 & 0 & 0 & 5 & 0 & 0 & 0 & 0 \\
\hline 0 & 13 & 0 & 0 & 0 & 3 & 0 & 14 & 0 & 0 \\
\hline 0 & 14 & 0 & 1 & 0 & 0 & 0 & 0 & 0 & 3 \\
\hline 0 & 0 & 0 & 0 & 0 & 0 & 0 & 150 & 0 & 3 \\
\hline 0 & 7 & 0 & 0 & 0 & 0 & 0 & 173 & 0 & 0 \\
\hline 0 & 5 & 0 & 0 & 0 & 0 & 0 & 0 & 0 & 4 \\
\hline 0 & 0 & 0 & 0 & 0 & 0 & 0 & 156 & 0 & 31 \\
\hline 0 & 10 & 0 & 0 & 0 & 0 & 0 & 337 & 0 & 4 \\
\hline 0 & 0 & 0 & 0 & 0 & 0 & 0 & 15 & 0 & 0 \\
\hline 0 & 9 & 0 & 0 & 0 & 0 & 0 & 8 & 0 & 0 \\
\hline 0 & 14 & 0 & 0 & 0 & 0 & 0 & 0 & 0 & 0 \\
\hline 0 & 0 & 0 & 0 & 0 & 0 & 0 & 70 & 0 & 0 \\
\hline 0 & 3 & 0 & 0 & 0 & 0 & 0 & 90 & 0 & 0 \\
\hline 0 & 0 & 0 & 12 & 0 & 2 & 0 & 0 & 0 & 0 \\
\hline 0 & 0 & 0 & 0 & 0 & 9 & 0 & 288 & 0 & 0 \\
\hline 0 & 0 & 0 & 18 & 0 & 0 & 0 & 130 & 0 & 0 \\
\hline 0 & 0 & 0 & 21 & 0 & 3 & 0 & 7 & 0 & 2 \\
\hline 0 & 0 & 0 & 0 & 0 & 10 & 0 & 0 & 0 & 0 \\
\hline 0 & 0 & 0 & 12 & 0 & 0 & 0 & 0 & 0 & 8 \\
\hline 0 & 0 & 0 & 7 & 0 & 0 & 0 & 5 & 0 & 20 \\
\hline 0 & 0 & 0 & 0 & 0 & 0 & 0 & 0 & 0 & 0 \\
\hline 0 & 0 & 0 & 6 & 0 & 0 & 0 & 0 & 0 & 82 \\
\hline 0 & 0 & 0 & 0 & 0 & 0 & 0 & 3 & 0 & 99 \\
\hline 0 & 8 & 0 & 22 & 0 & 0 & 0 & 0 & 0 & 69 \\
\hline 0 & 0 & 0 & 13 & 0 & 0 & 0 & 0 & 0 & 0 \\
\hline 0 & 62 & 0 & 0 & 0 & 0 & 0 & 0 & 0 & 0 \\
\hline 0 & 65 & 0 & 11 & 0 & 0 & 0 & 0 & 0 & 10 \\
\hline 0 & 0 & 0 & 29 & 0 & 42 & 0 & 0 & 0 & 13 \\
\hline 0 & 17 & 0 & 0 & 0 & 0 & 0 & 0 & 0 & 0 \\
\hline 0 & 9 & 0 & 0 & 0 & 69 & 0 & 0 & 1986 & 88 \\
\hline 0 & 0 & 0 & 0 & 0 & 117 & 0 & 0 & 0 & 1580 \\
\hline 0 & 3 & 0 & 0 & 0 & 0 & 0 & 0 & 0 & 233 \\
\hline 0 & 0 & 0 & 0 & 0 & 0 & 0 & 0 & 0 & 0 \\
\hline 0 & 0 & 0 & 0 & 0 & 0 & 0 & 0 & 0 & 61 \\
\hline 0 & 0 & 0 & 0 & 0 & 0 & 0 & 0 & 0 & 0 \\
\hline 0 & 0 & 0 & 0 & 0 & 0 & 0 & 0 & 0 & 0 \\
\hline 0 & 0 & 0 & 0 & 0 & 0 & 0 & 0 & 0 & 0 \\
\hline 0 & 0 & 0 & 0 & 0 & 0 & 0 & 0 & 0 & 0 \\
\hline 0 & 0 & 0 & 0 & 0 & 0 & 0 & 0 & 38015 & 34684 \\
\hline 0 & 427 & 0 & 205 & 0 & 413 & 0 & 1950 & 40001 & 37006 \\
\hline
\end{tabular}

C-Scan image for reference and damaged composite

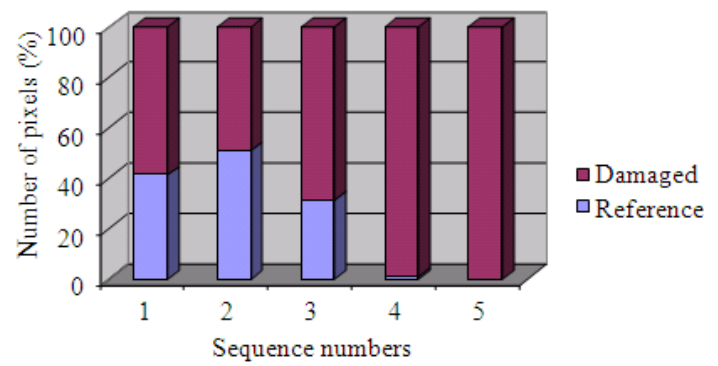

Fig. 6: Extent of damage in a $5 \mathrm{~mm}$ woven glass using C-Scan and ICA
LTT image for reference and damaged composite

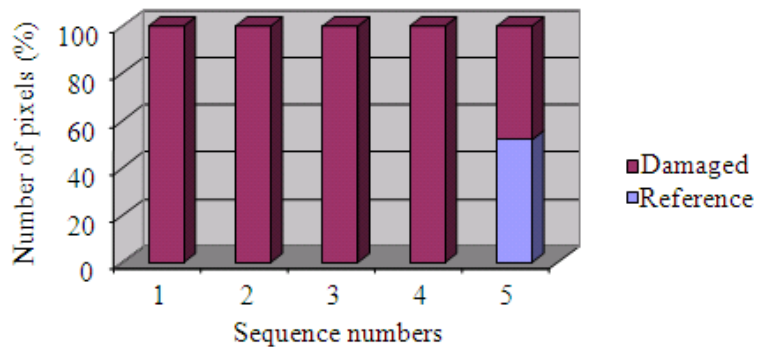

Fig. 7: Extent of damage in a $5 \mathrm{~mm}$ woven glass using LTT and ICA 
J. Computer Sci., 6 (4): 428-438, 2010

Table 3: C-Scan and LTT reference sequences for $5 \mathrm{~mm}$ woven glass

\begin{tabular}{|c|c|c|c|c|c|c|c|c|c|}
\hline$\underline{S_{1 \text { Ref CScan }}}$ & $S_{1 \text { Ref LTT }}$ & $S_{2 \text { Ref CScan }}$ & $\mathrm{S}_{2 \text { Ref LTT }}$ & $S_{3 \text { Ref CScan }}$ & $\mathrm{S}_{3 \text { Ref LTT }}$ & $S_{4 \text { Ref CScan }}$ & $\mathrm{S}_{4 \text { Ref LTT }}$ & $\mathrm{S}_{5 \text { Ref CScan }}$ & $S_{5 \text { Ref LTT }}$ \\
\hline 0 & 0 & 0 & 0 & 0 & 0 & 0 & 0 & 0 & 0 \\
\hline 0 & 0 & 0 & 0 & 0 & 0 & 0 & 0 & 0 & 0 \\
\hline 0 & 0 & 1250 & 0 & 0 & 0 & 0 & 0 & 0 & 0 \\
\hline 0 & 0 & 0 & 0 & 0 & 0 & 0 & 0 & 0 & 0 \\
\hline 578 & 0 & 0 & 0 & 0 & 0 & 1 & 0 & 0 & 0 \\
\hline 0 & 0 & 0 & 0 & 0 & 0 & 0 & 0 & 0 & 0 \\
\hline 0 & 0 & 0 & 0 & 0 & 0 & 0 & 0 & 0 & 0 \\
\hline 0 & 0 & 1 & 0 & 0 & 0 & 0 & 0 & 0 & 0 \\
\hline 0 & 0 & 0 & 0 & 0 & 0 & 0 & 0 & 0 & 0 \\
\hline 0 & 0 & 0 & 0 & 0 & 0 & 0 & 0 & 0 & 0 \\
\hline 0 & 0 & 0 & 0 & 0 & 0 & 0 & 0 & 0 & 0 \\
\hline 0 & 0 & 0 & 0 & 0 & 0 & 0 & 0 & 0 & 0 \\
\hline 0 & 0 & 0 & 0 & 0 & 0 & 0 & 0 & 0 & 0 \\
\hline 0 & 0 & 0 & 0 & 0 & 0 & 0 & 0 & 0 & 0 \\
\hline 0 & 0 & 0 & 0 & 0 & 0 & 0 & 0 & 0 & 0 \\
\hline 0 & 0 & 0 & 0 & 0 & 0 & 0 & 0 & 0 & 0 \\
\hline 0 & 0 & 0 & 0 & 0 & 0 & 0 & 0 & 0 & 0 \\
\hline 0 & 0 & 0 & 0 & 0 & 0 & 0 & 0 & 0 & 0 \\
\hline 0 & 0 & 26969 & 0 & 0 & 0 & 0 & 0 & 0 & 0 \\
\hline 0 & 0 & 0 & 0 & 0 & 0 & 0 & 0 & 0 & 0 \\
\hline 0 & 0 & 0 & 0 & 0 & 0 & 0 & 0 & 0 & 0 \\
\hline 0 & 0 & 0 & 0 & 0 & 0 & 0 & 0 & 0 & 0 \\
\hline 0 & 0 & 0 & 0 & 0 & 0 & 0 & 0 & 0 & 0 \\
\hline 0 & 0 & 0 & 0 & 0 & 0 & 0 & 0 & 0 & 0 \\
\hline 0 & 0 & 2 & 0 & 0 & 0 & 0 & 0 & 0 & 0 \\
\hline 0 & 0 & 0 & 0 & 0 & 0 & 0 & 0 & 0 & 0 \\
\hline 0 & 0 & 10102 & 0 & 0 & 0 & 0 & 0 & 0 & 0 \\
\hline 0 & 0 & 0 & 0 & 0 & 0 & 0 & 0 & 0 & 0 \\
\hline 0 & 0 & 0 & 0 & 0 & 0 & 0 & 0 & 0 & 0 \\
\hline 0 & 0 & 0 & 0 & 0 & 0 & 0 & 0 & 0 & 0 \\
\hline 1 & 0 & 0 & 0 & 0 & 0 & 0 & 0 & 0 & 0 \\
\hline 0 & 0 & 0 & 0 & 0 & 0 & 0 & 0 & 0 & 0 \\
\hline 0 & 0 & 0 & 0 & 0 & 0 & 0 & 0 & 0 & 0 \\
\hline 0 & 0 & 0 & 0 & 0 & 0 & 0 & 0 & 0 & 0 \\
\hline 0 & 0 & 152 & 0 & 1 & 0 & 0 & 0 & 0 & 0 \\
\hline 0 & 0 & 0 & 0 & 0 & 0 & 0 & 0 & 0 & 0 \\
\hline 0 & 0 & 0 & 0 & 0 & 0 & 0 & 0 & 0 & 0 \\
\hline 0 & 0 & 0 & 0 & 0 & 0 & 0 & 0 & 0 & 0 \\
\hline 0 & 0 & 0 & 0 & 0 & 0 & 0 & 0 & 0 & 0 \\
\hline 0 & 0 & 0 & 0 & 0 & 0 & 0 & 0 & 0 & 0 \\
\hline 0 & 0 & 0 & 0 & 0 & 0 & 0 & 0 & 0 & 0 \\
\hline 0 & 0 & 0 & 0 & 0 & 0 & 0 & 0 & 0 & 0 \\
\hline 0 & 0 & 0 & 0 & 0 & 0 & 0 & 0 & 0 & 0 \\
\hline 1 & 0 & 0 & 0 & 0 & 0 & 0 & 0 & 0 & 0 \\
\hline 0 & 0 & 0 & 0 & 943 & 0 & 0 & 0 & 0 & 0 \\
\hline 0 & 0 & 0 & 0 & 0 & 0 & 0 & 0 & 0 & 0 \\
\hline 0 & 0 & 0 & 0 & 0 & 0 & 0 & 0 & 0 & 1986 \\
\hline 0 & 0 & 0 & 0 & 0 & 0 & 0 & 0 & 0 & 0 \\
\hline 0 & 0 & 0 & 0 & 0 & 0 & 0 & 0 & 0 & 0 \\
\hline 0 & 0 & 0 & 0 & 0 & 0 & 0 & 0 & 0 & 0 \\
\hline 0 & 0 & 0 & 0 & 0 & 0 & 0 & 0 & 0 & 0 \\
\hline 0 & 0 & 0 & 0 & 0 & 0 & 0 & 0 & 0 & 0 \\
\hline 0 & 0 & 0 & 0 & 0 & 0 & 0 & 0 & 0 & 0 \\
\hline 0 & 0 & 0 & 0 & 0 & 0 & 0 & 0 & 0 & 0 \\
\hline 0 & 0 & 0 & 0 & 0 & 0 & 0 & 0 & 0 & 0 \\
\hline 0 & 0 & 0 & 0 & 0 & 0 & 0 & 0 & 0 & 38015 \\
\hline 580 & 0 & 38476 & 0 & 944 & 0 & 1 & 0 & 0 & 40001 \\
\hline
\end{tabular}

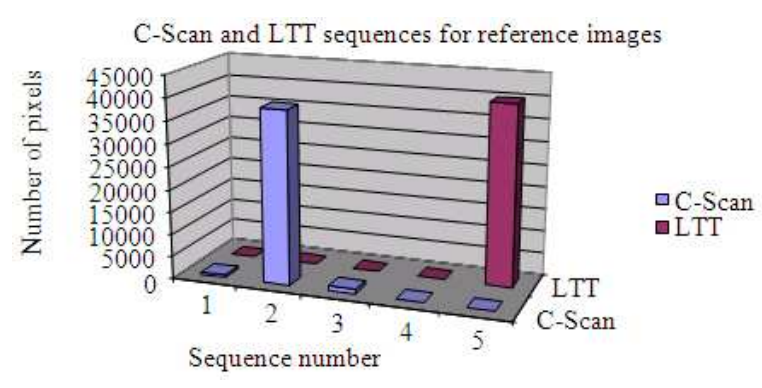

Fig. 8: Relationship between sequences in C-Scan and LTT Pre-impact images of $5 \mathrm{~mm}$ woven glass

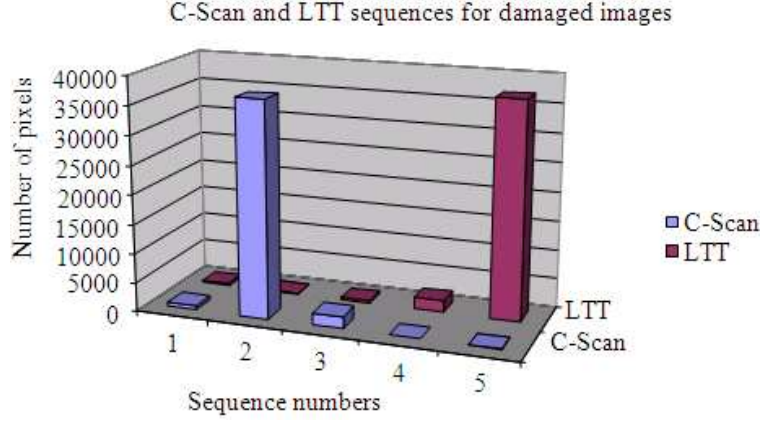

Fig. 9: Relationship between sequences in C-Scan and LTT post-impact images of $5 \mathrm{~mm}$ woven glass 
J. Computer Sci., 6 (4): 428-438, 2010

Table 4: C-Scan and LTT reference sequences for $5 \mathrm{~mm}$ woven glass

\begin{tabular}{|c|c|c|c|c|c|c|c|c|c|}
\hline $\mathrm{S}_{1 \text { Dam LTT }}$ & $\mathrm{S}_{1 \text { Dam CScan }}$ & $\mathrm{S}_{2 \text { Dam LTT }}$ & $\mathrm{S}_{2 \text { Dam CScan }}$ & $\mathrm{S}_{3 \text { Dam LTT }}$ & $\mathrm{S}_{3 \text { Dam CScan }}$ & $\mathrm{S}_{4 \text { Dam LTT }}$ & $\mathrm{S}_{4 \text { Dam CScan }}$ & $\mathrm{S}_{5 \text { Dam LTT }}$ & $\mathrm{S}_{5 \text { Dam CScan }}$ \\
\hline 0 & 7 & 26 & 37 & 0 & 0 & 47 & 8 & 0 & 0 \\
\hline 4 & 21 & 16 & 14 & 0 & 0 & 7 & 1 & 0 & 0 \\
\hline 0 & 0 & 0 & 2831 & 0 & 0 & 27 & 0 & 0 & 0 \\
\hline 0 & 0 & 1 & 0 & 0 & 0 & 0 & 2 & 0 & 0 \\
\hline 19 & 623 & 2 & 32 & 2 & 2 & 122 & 4 & 0 & 0 \\
\hline 0 & 0 & 0 & 36 & 0 & 0 & 98 & 1 & 0 & 0 \\
\hline 0 & 0 & 0 & 0 & 32 & 6 & 0 & 0 & 0 & 0 \\
\hline 73 & 1 & 0 & 21 & 39 & 6 & 94 & 0 & 0 & 0 \\
\hline 29 & 1 & 0 & 19 & 0 & 0 & 103 & 5 & 0 & 12 \\
\hline 58 & 1 & 0 & 12 & 29 & 5 & 6 & 0 & 0 & 0 \\
\hline 0 & 7 & 0 & 0 & 18 & 4 & 0 & 0 & 0 & 1 \\
\hline 0 & 0 & 0 & 0 & 1 & 1 & 0 & 3 & 0 & 1 \\
\hline 0 & 0 & 0 & 0 & 3 & 4 & 0 & 5 & 5 & 0 \\
\hline 0 & 0 & 0 & 0 & 0 & 0 & 0 & 0 & 0 & 0 \\
\hline 1 & 10 & 7 & 0 & 21 & 5 & 0 & 0 & 2 & 1 \\
\hline 0 & 0 & 0 & 0 & 8 & 6 & 0 & 0 & 4 & 0 \\
\hline 12 & 12 & 1 & 0 & 0 & 0 & 0 & 4 & 1 & 21 \\
\hline 0 & 0 & 0 & 0 & 5 & 2 & 0 & 1 & 0 & 0 \\
\hline 13 & 6 & 0 & 23306 & 3 & 4 & 14 & 0 & 0 & 0 \\
\hline 14 & 11 & 1 & 0 & 0 & 1 & 0 & 0 & 3 & 0 \\
\hline 0 & 0 & 0 & 172 & 0 & 2 & 150 & 5 & 3 & 0 \\
\hline 7 & 17 & 0 & 125 & 0 & 0 & 173 & 8 & 0 & 0 \\
\hline 5 & 39 & 0 & 0 & 0 & 4 & 0 & 0 & 4 & 1 \\
\hline 0 & 10 & 0 & 24 & 0 & 0 & 156 & 0 & 31 & 0 \\
\hline 10 & 3 & 0 & 18 & 0 & 0 & 337 & 0 & 4 & 0 \\
\hline 0 & 0 & 0 & 10 & 0 & 1 & 15 & 0 & 0 & 0 \\
\hline 9 & 8 & 0 & 8785 & 0 & 5 & 8 & 0 & 0 & 0 \\
\hline 14 & 4 & 0 & 0 & 0 & 2 & 0 & 0 & 0 & 0 \\
\hline 0 & 0 & 0 & 129 & 0 & 0 & 70 & 0 & 0 & 0 \\
\hline 3 & 10 & 0 & 61 & 0 & 0 & 90 & 0 & 0 & 0 \\
\hline 0 & 10 & 12 & 0 & 2 & 23 & 0 & 1 & 0 & 0 \\
\hline 0 & 5 & 0 & 25 & 9 & 12 & 288 & 0 & 0 & 0 \\
\hline 0 & 0 & 18 & 14 & 0 & 0 & 130 & 5 & 0 & 14 \\
\hline 0 & 0 & 21 & 2 & 3 & 3 & 7 & 4 & 2 & 0 \\
\hline 0 & 0 & 0 & 1073 & 10 & 14 & 0 & 0 & 0 & 0 \\
\hline 0 & 0 & 12 & 0 & 0 & 14 & 0 & 7 & 8 & 7 \\
\hline 0 & 0 & 7 & 21 & 0 & 0 & 5 & 19 & 20 & 0 \\
\hline 0 & 0 & 0 & 14 & 0 & 0 & 0 & 9 & 0 & 0 \\
\hline 0 & 0 & 6 & 0 & 0 & 0 & 0 & 2 & 82 & 6 \\
\hline 0 & 0 & 0 & 2 & 0 & 0 & 3 & 0 & 99 & 4 \\
\hline 8 & 1 & 22 & 2 & 0 & 0 & 0 & 6 & 69 & 5 \\
\hline 0 & 0 & 13 & 6 & 0 & 0 & 0 & 8 & 0 & 0 \\
\hline 62 & 3 & 0 & 1 & 0 & 0 & 0 & 0 & 0 & 2 \\
\hline 65 & 2 & 11 & 0 & 0 & 0 & 0 & 4 & 10 & 0 \\
\hline 0 & 0 & 29 & 0 & 42 & 1906 & 0 & 2 & 13 & 0 \\
\hline 17 & 2 & 0 & 0 & 0 & 0 & 0 & 6 & 0 & 1 \\
\hline 9 & 2 & 0 & 0 & 69 & 27 & 0 & 0 & 88 & 1 \\
\hline 0 & 4 & 0 & 0 & 117 & 21 & 0 & 0 & 1580 & 23 \\
\hline 3 & 5 & 0 & 1 & 0 & 0 & 0 & 0 & 233 & 0 \\
\hline 0 & 0 & 0 & 1 & 0 & 0 & 0 & 0 & 0 & 18 \\
\hline 0 & 0 & 0 & 0 & 0 & 0 & 0 & 0 & 61 & 37 \\
\hline 0 & 0 & 0 & 0 & 0 & 0 & 0 & 0 & 0 & 7 \\
\hline 0 & 0 & 0 & 0 & 0 & 0 & 0 & 0 & 0 & 2 \\
\hline 0 & 0 & 0 & 0 & 0 & 0 & 0 & 0 & 0 & 2 \\
\hline 0 & 0 & 0 & 0 & 0 & 0 & 0 & 0 & 0 & 11 \\
\hline 0 & 0 & 0 & 0 & 0 & 0 & 0 & 0 & 34684 & 5 \\
\hline 427 & 825 & 205 & 36794 & 413 & 2080 & 1950 & 120 & 37006 & 182 \\
\hline
\end{tabular}

- Figure 6 show the extent of damage occurred by $28.6 \mathrm{~J}$ to the $5 \mathrm{~mm}$ thick woven glass component when tested by C-Scan and LTT. The LTT technique and image contained better information regarding the severity of damage compared to the C-Scan one

- The developed classification algorithm provided clear and accurate decision regarding component usability 
with acceptability tolerance values. Such algorithm is novel with its unique feature of determining the required margin for composite structure application before a components deemed unusable

- Same size images used for both C-Scan and LTT to enable correct comparison and correlation

- The ICA algorithm is based on the Energy Band Model, which assumes that image pixel values and distribution in energy levels is modified and modulated due to impact damage. Such energy level change is a function of the Impact energy and tensile strength of the material.

Applying derived expressions to experimental images gives:

$$
\mathrm{D}_{\text {Ref (C-Scan) }}=\left[\begin{array}{c}
00580 \\
38476 \\
00944 \\
00001 \\
00000
\end{array}\right]
$$$$
\mathrm{D}_{\text {Dam(C-Scan) }}=\left[\begin{array}{c}
00825 \\
36794 \\
02080 \\
00182 \\
00120
\end{array}\right]
$$

$$
\left.P_{(\mathrm{C}-\mathrm{Scan}}\right)=\left[\begin{array}{c}
00245 \\
01682 \\
01136 \\
00181 \\
00120
\end{array}\right]
$$

$$
\mathrm{D}_{\text {Ref(LTT) }}=\left[\begin{array}{c}
00000 \\
00000 \\
00000 \\
00000 \\
40001
\end{array}\right]
$$

$$
\mathrm{D}_{\text {Dam(LTT) }}=\left[\begin{array}{c}
00427 \\
00205 \\
00413 \\
01950 \\
37006
\end{array}\right]
$$

$$
\mathrm{P}_{\text {(LTT) }}=\left[\begin{array}{c}
00427 \\
00205 \\
00413 \\
01950 \\
02995
\end{array}\right]
$$

The classification matrix Percentage tolerance values are given by:

$$
\left.\mathrm{F}_{(\mathrm{C}-\mathrm{Scan}}\right)=\left[\begin{array}{c}
0.600 \\
4.200 \\
2.800 \\
0.450 \\
0.300
\end{array}\right]
$$

$$
\mathrm{F}_{(\mathrm{LTT})}=\left[\begin{array}{c}
1.100 \\
0.500 \\
1.000 \\
4.900 \\
7.500
\end{array}\right]
$$

For $1-4 \%$ damage tolerance, C-Scan technique judge the component as damaged. For 1-7\%, LTT technique judges the component damaged. Such values and others related to different testing techniques are associated with their respective testing data and type of composite structure in a database that is used in conjunction with the ICA algorithm as an expert system for missing data classification and damage level prediction. The classification matrix percentage tolerance can be combined in a hybrid matrix giving:

$$
\mathrm{F}_{\text {Hybrid }}=\left[\begin{array}{cc}
0.600 & 1.100 \\
4.200 & 0.500 \\
2.800 & 1.000 \\
0.450 & 4.900 \\
0.300 & 7.500
\end{array}\right]
$$

The Hybrid Matrix can house as many testing techniques as required with ability for matrix manipulation to be carried out to consolidate ICA decision making.

\section{CONCLUSION}

The developed ICA algorithm unifies through conversion the extracted information from irrelevant background with ability to correlate obtained data to level of damage and it is effect on the structure overall 
performance. Also, the known difficulty in the interpretation of the damage data is solved through a specialized analysis and interpretation algorithm (MCA) specifically developed to indicate the severity of the damage and its effect on the general performance of the component. This very successful approach to damage detection and classification is further supported by its ability to correlate different NDT technologies and predict others.

\section{REFERENCES}

Bohm, S., M. Hellmanns, A. Backes and K. Dilger, 2006. Lock-In thermography based NDT of parts for the automotive industry. J. Adhesion Interface, 7: $10-12$.

http://www.adhesioninterface.org/journal/0704/02W3.pdf

Breitzman, T., E. Iarve, B. Cook, G. Schoeppner and R. Lipton, 2009. Optimization of a composite scarf repair patch under tensile loading. Composites Part A: Applied Sci. Manufact., 40: 1921-1930. DOI: 10.1016/j.compositesa.2009.04.033

Chinnam, R. and P. Baruah, 2007. Empirical prediction limit estimation methods for feed-forward neural networks. Int. J. Gen. Syst., 36: 221-236. DOI: 10.1080/03081070600782170

Colvin, J.B., 2005. Moire stabilized thermal imaging. Proceedings of the 12th IPFA, IEEE, Singapore, pp: 163-166.

http://www.fainstruments.com/PDF/IFPA2005Moire.pdf

Eklund, N. and K. Goebel, 2005. using neural networks and the rank permutation transformation to detect abnormal conditions in aircraft engines. Proceeding of the IEEE Mid-Summer Workshop on Soft Computing in Industrial Applications, June 28-30, Helsinki University of Technology, Espoo, Finland, pp: 1-5. http://www.alliedchennai.com/ieeecf2005.html

Goebel, K., W. Yan, N. Eklund and X. Hu, 2006a. Defect classification of highly noisy NDE data using classifier ensembles. Proceedings of the SPIE; Smart Structures and Materials 2006: Smart Sensor Monitor. Syst. Applications, pp: 460-471. http://best.berkeley.edu/ goebel/publications_files/ SPIE06_1_r.pdf

Goebel, K., X. Hu, N. Eklund and W. Yan, 2006b. Fusing diverse monitoring algorithms for robust change detection, Proceedings of the SPIE, Multisensor, Multisource Information Fusion: Architectures, Algorithms Applications, pp: 192-202. http://www.geglobalresearch.com/ServiceAlgorith $\mathrm{ms} / \mathrm{html} /$ publications/pdf/37.pdf
Gralewicz, G. and G. Owczarek, 2005. Investigations of single and multilayer structures using lock-in thermography-possible applications. Int. J. Occupat. Safe. Ergon., 11: 211-215. http://www.ciop.pl/13341

Gupta, R. and O. Breitenstein, 2007. Unsteady-state lock-in thermography-Application to shunts in solar cells. QIRT J., 4: 85-105. http://www.mpihalle.de/mpi/publi/pdf/7456_07.pdf

Hayman, B., 2007. Approaches to damage assessment and damage tolerance for FRP sandwich structures. J. Sandwich Struct. Mater., 9: 571-596. DOI: 10.1177/1099636207070853

Hu, X., N. Eklund and K. Goebel, 2006. Using rank permutation for aircraft engine fault diagnostics. Proceeding of the 60th Meeting of the Society For Machinery Failure Prevention Technology (MFPT), pp: 396-403. http://best.berkeley.edu/ goebel/publications_files/ MFPT_2_r.pdf

Jenab, K. and K. Rashidi, 2009. Fuzzy Bayesian condition monitoring model based on exponential distribution. Int. J. Eng. Technol., 1: 172-178. DOI: 10.1080/00207540802232930

Kim, J., F. Pierron, M. Wisnom and S. Avril, 2009. Local stiffness reduction in impacted composite plates from full-field measurements. Composites Part A: Applied Sci. Manufact., 40: 1961-1974. DOI: 10.1016/j.compositesa.2009.09.024

Li, X., S. Hallett, M. Wisnom and N. Zobeiry et al., 2009. Experimental study of damage propagation in over-height compact tension tests. Composites Part A: Applied Sci. Manufact., 40: 1891-1899. DOI:10.1016/j.compositesa.2009.08.017

Montanini, R. and S. Aliquo, 2009. nondestructive evaluation of plexiglas materials using lock-in and pulse phase infrared thermography. Proceedings of the 19th IMEKO World Congress: Fundamental and Applied Metrology, Sept. 6-11, LisbonPortugal, pp: 1524-1529. http://www.imeko.org/publications/wc2009/IMEKO-WC-2009-TC12-220.pdf

Mouritz, A., S. Feih, E. Kandare, Z. Mathys and A.G. Gibson et al., 2009. Review of fire structural modelling of polymer composites. Composites Part A: Applied Sci. Manufact., 40: 1800-1814. DOI: 10.1016/j.compositesa.2009.09.001

Shah, J., S. Aglyamov, K. Sokolov, T. Milner and S. Emelianov, 2006. Ultrasound-based thermal and elasticity imaging to assist photothermal cancer therapy-preliminary study. Proceedings of the IEEE Ultrasonics Symposium, IEEE, pp: 1029-1032. http://www.bme.utexas.edu/research/pil/papers/Sha h_IEEE_US_2006.pdf 
Stoika, C., M. Bohnb and J. Blackshire, 2009. Nondestructive evaluation of aircraft composites using reflective terahertz time domain spectroscopy. NDT E Int., 43: 106-115. DOI: 10.1016/j.ndteint.2009.09.005

Tohgo, K., Y. Itoh and Y. Shimamura, 2009. A constitutive model of particulate-reinforced composites taking account of particle size effects and damage evolution. Comp. Part A: Applied Sci. Manufactur., $\quad 41$ : 313-320. $\quad$ DOI: 10.1016/j.compositesa.2009.10.023

Verdegaya, J., R. Yager and P. Bonissone, 2008. On heuristics as a fundamental constituent of soft computing. Fuzzy Sets Syst., 159: 846-855. DOI: $10.1016 /$ j.fss.2007.08.014

Williams, H., R. Trask and I. Bond, 2008. Self-healing sandwich panels: Restoration of compressive strength after impact. Compos. Sci. Technol., 68: 3171-3177. 10.1016/j.compscitech.2008.07.016
Wolf, A., P. Pohl and R. Brendel, 2004. Thermophysical analysis of thin films by lock-in thermography. J. Applied Phys., 96: 6306-6312. http://www.zae-bayern.de/files/pub_2004_09.pdf

Zangani, D., M. Robinson, A. Gibson, L. Torre and A. Holmberg, 2007. Numerical simulation of bending and failure behaviour of z-core sandwich panels. Plastics Rubber and Compos., 36: 389-395. DOI: 10.1179/174328907X248195

Zhang, Z. and M. Richardson, 2004. Visualization of barely visible impact damage in polymer matrix composites using an Optical Deformation and Strain Measurement System (ODSMS). Composites: Part A, 36: 1073-1078. DOI: 10.1016/j.compositesa.2004.10.035 